\title{
Shannon capacity and the categorical product
}

\author{
Gábor Simonyi* \\ Alfréd Rényi Institute of Mathematics, Budapest, Hungary \\ and \\ Department of Computer Science and Information Theory, \\ Budapest University of Technology and Economics \\ simonyi@renyi.hu
}

Submitted: Oct 31, 2019; Accepted: Feb 16, 2021; Published: Mar 12, 2021

(C) The author. Released under the CC BY-ND license (International 4.0).

\begin{abstract}
Shannon OR-capacity $C_{\mathrm{OR}}(G)$ of a graph $G$, that is the traditionally more often used Shannon AND-capacity of the complementary graph, is a homomorphism monotone graph parameter therefore $C_{\mathrm{OR}}(F \times G) \leqslant \min \left\{C_{\mathrm{OR}}(F), C_{\mathrm{OR}}(G)\right\}$ holds for every pair of graphs, where $F \times G$ is the categorical product of graphs $F$ and $G$. Here we initiate the study of the question when could we expect equality in this inequality. Using a strong recent result of Zuiddam, we show that if this "Hedetniemitype" equality is not satisfied for some pair of graphs then the analogous equality is also not satisfied for this graph pair by some other graph invariant that has a much "nicer" behavior concerning some different graph operations. In particular, unlike Shannon OR-capacity or the chromatic number, this other invariant is both multiplicative under the OR-product and additive under the join operation, while it is also nondecreasing along graph homomorphisms. We also present a natural lower bound on $C_{\mathrm{OR}}(F \times G)$ and elaborate on the question of how to find graph pairs for which it is known to be strictly less than the upper bound $\min \left\{C_{\mathrm{OR}}(F), C_{\mathrm{OR}}(G)\right\}$. We present such graph pairs using the properties of Paley graphs.
\end{abstract}

Mathematics Subject Classifications: 05C15, 05C76

\section{Introduction}

For two graphs $F$ and $G$, their categorical (also called tensor or weak direct) product $F \times G$ is defined by

$$
V(F \times G)=V(F) \times V(G),
$$

*Research partially supported by the National Research, Development and Innovation Office (NKFIH) grants K-116769, K-120706, K-132696, SSN-135643, by the BME NC TKP2020 grant of NKFIH Hungary and by the BME-Artificial Intelligence FIKP grant of EMMI (BME FIKP-MI/SC). 


$$
E(F \times G)=\{\{(x, u),(y, v)\}: x, y \in V(F), u, v \in V(G),\{x, y\} \in E(F),\{u, v\} \in E(G)\} .
$$

Hedetniemi's more than half a century old conjecture that has been refuted recently by Yaroslav Shitov [44] (cf. also [52], [24], [58], [59], [50], and [53] for further developments) stated that the chromatic number of $F \times G$ would be equal to the smaller of the chromatic numbers of $F$ and $G$, i.e., that

$$
\chi(F \times G)=\min \{\chi(F), \chi(G)\} .
$$

It is easy to see that the right hand side above is an upper bound on the left hand side. If $c: V(F) \rightarrow\{1,2, \ldots, \chi(F)\}$ is a proper coloring of $F$ then $c^{\prime}:(x, u) \mapsto c(x)$ is a proper coloring of $F \times G$ proving $\chi(F \times G) \leqslant \chi(F)$. As the same argument works if we start with a proper coloring of $G$, this proves the claimed inequality. Thus the real content of the conjecture was that the right hand side is also a lower bound on $\chi(F \times G)$. Though not true in general, this holds in several special cases. In particular, it is easy to prove when $\min \{\chi(F), \chi(G)\} \leqslant 3$ and it is also known to hold when this value is 4 . The latter, however, is a highly nontrivial result of El-Zahar and Sauer [15] and the general case was wide open until the already mentioned recent breakthrough by Shitov [44]. For several related results, see the survey papers $[41,49,56]$.

A map $f: V(T) \rightarrow V(H)$ between the vertex sets of graphs $T$ and $H$ is called a graph homomorphism if it preserves edges, that is, if $f$ satisfies $\{a, b\} \in E(T) \Rightarrow\{f(a), f(b)\} \in$ $E(H)$. The existence of a graph homomorphism from $T$ to $H$ is denoted by $T \rightarrow H$. Recall that a proper coloring of a graph $G$ with $n$ colors is nothing but a homomorphism from $G$ to the complete graph on $n$ vertices.

Behind the validity of the inequality $\chi(F \times G) \leqslant \min \{\chi(F), \chi(G)\}$ is the fact that both $F \times G \rightarrow F$ and $F \times G \rightarrow G$ hold (just take the projection maps), while it is generally true, that $T \rightarrow H$ implies $\chi(T) \leqslant \chi(H)$.

This suggests that if $p(G)$ is any graph parameter which is monotone nondecreasing under graph homomorphism, that is for which $T \rightarrow H$ implies $p(T) \leqslant p(H)$, then an analogous question to Hedetniemi's conjecture is meaningful for it: we automatically have $p(F \times G) \leqslant \min \{p(F), p(G)\}$ and one may ask whether equality holds. (If it does, we will say that $p$ satisfies the Hedetniemi-type equality.) Since Shitov's result we know that the most studied parameter from this point of view, the chromatic number, does not satisfy the Hedetniemi-type equality. But there are parameters that do so. A trivial example is provided by the clique number. Two important parameters for which the corresponding equality non-trivially holds are treated by theorems of Zhu [57] and Godsil, Roberson, Šamal, and Severini [19].

Zhu's celebrated result known as the fractional version of Hedetniemi's conjecture [57] states that the fractional chromatic number satisfies the Hedetniemi-type equality. (For the definition and basic properties of the fractional chromatic number, see e.g. [42].) The more recent result by Godsil, Roberson, Šamal, and Severini [19] is that the similar statement is also true for the Lovász theta number of the complementary graph, which is also known as the strict vector chromatic number. (For the definition and basic properties 
of this parameter see Lovász's original paper [33] or his recent book [35].) In [19] the authors also investigated the analogous question for a closely related parameter called vector chromatic number (which differs from the strict vector chromatic number) that was introduced in [29]. They conjectured that the Hedetniemi-type equality also holds for this parameter and proved it in special cases. In a follow-up paper by Godsil, Roberson, Roomey, Šamal, and Varvitsiotis [20] the latter conjecture is proved in general as well.

Both the fractional chromatic number and the Lovász theta number of the complementary graph are well-known upper bounds on the Shannon OR-capacity of the graph which is the usual Shannon capacity, or Shannon AND-capacity of the complementary graph. This is also a homomorphism-monotone parameter (see Proposition 3 in Section 2), so the Hedetniemi-type question is meaningful for it, too. In this paper we initiate the study of this question. That is, we are interested in conditions on graphs $F$ and $G$ that make the equality

$$
C_{\mathrm{OR}}(F \times G)=\min \left\{C_{\mathrm{OR}}(F), C_{\mathrm{OR}}(G)\right\}
$$

true. In particular, we ask whether this Hedetniemi-type equality might hold for all graph pairs.

Given the very complex behavior of Shannon capacity there seems to be little reason to believe that the Shannon OR-capacity would also satisfy the Hedetniemi-type equality as the above mentioned two graph parameters do. However, if one has to argue why it looks unlikely, then the first argument that comes to mind is that Shannon OR-capacity famously does not satisfy two other simple equalities, namely it is not multiplicative under the OR-product and not additive under graph join. (For the definition of these graph operations see Definition 1 in Section 2 and Definition 5 in Section 3, respectively.) In both cases, this is a non-trivial result. Whether the first mentioned multiplicativity relation holds was asked by Lovász in [33] and answered in the negative by Haemers [22] by introducing a new upper bound on Shannon capacity that sometimes beats the Lovász theta number. The second mentioned additivity relation was conjectured to hold by Shannon [43] and this conjecture has been refuted only more than forty years later by Alon [1].

One has to add that there are graph parameters, that (sometimes trivially) satisfy the Hedetniemi-type equality, while they do not satisfy (at least one of) the other two equalities mentioned above (cf. Remarks 2 and 3 in Section 3), yet in those cases we know about the behaviour of the parameters involved is less complex than that of Shannon capacity. (Note that even the decidability of the computational problem given by Shannon capacity is not known, cf. [2].)

On the other hand, both the fractional chromatic number and the Lovász theta number of the complementary graph are multiplicative with respect to the OR-product and additive under graph join. They both belong to a family of graph parameters that behave particularly "nicely" and every member of which, as a real valued parameter of a graph $G$, provides an upper bound on the Shannon OR-capacity of $G$. (For the precise definition of this family of graph parameters, called the asymptotic spectrum of graphs in [60], see Definition 6 in Section 3.) One may easily have the intuition that perhaps all parame- 
ters belonging to this family should satisfy the Hedetniemi-type equality, while Shannon OR-capacity should not. One of our main observations in this paper is that both of these intuitions cannot be correct at the same time: either there exists some graph parameter belonging to the asymptotic spectrum of graphs that does not satisfy the Hedetniemi-type equality, or the Hedetniemi-type equality should also hold for Shannon OR-capacity. This will be an almost immediate consequence of a strong recent result by Jeroen Zuiddam [60].

We will also elaborate on the question of finding graph pairs that provide potential counterexamples to Shannon OR-capacity satisfying the Hedetniemi-type equality. This turns out to be challenging as well, largely because of the lack of knowledge about the general behaviour of Shannon capacity. We will give a natural lower bound on $C_{\mathrm{OR}}(F \times G)$ in Section 4. If we want to "test" whether Shannon OR-capacity satisfies the Hedetniemitype equality in nontrivial cases, we need some graph pairs $(F, G)$, for which our lower bound is strictly smaller than the upper bound $\min \left\{C_{\mathrm{OR}}(F), C_{\mathrm{OR}}(G)\right\}$. Since the Shannon capacity value is known only in very few nontrivial cases, finding such graph pairs is not entirely trivial. We will present some graph pairs with this property in Subsection 4.3.

\section{Shannon OR-capacity}

The Shannon capacity of a graph involves a graph product which is different of the categorical product that appears in Hedetniemi's conjecture. In fact, traditionally, that is, in Shannon's original and in several subsequent other papers, see [43, 33, 22, 1, 9], it is defined via a product that is often called the AND-product (cf. e.g. [3]). Sometimes it is more convenient, however, to define graph capacity in a complementary way, cf. e.g. [12] (see Definition 11.3). The graph product involved then is the OR-product and the resulting notion is equivalent to the previous one defined for the complementary graph. To avoid confusion, we will call these two notions Shannon AND-capacity and Shannon OR-capacity, the latter being the one we will mostly use.

Definition 1. Let $F$ and $G$ be two graphs. Both their AND-product $F \otimes G$ and ORproduct $F \cdot G$ is defined on the Cartesian product $V(F) \times V(G)$ as vertex set. The edge set of the OR-product is given by

$$
\begin{gathered}
E(F \cdot G)= \\
\left\{\left\{(f, g),\left(f^{\prime}, g^{\prime}\right)\right\}: f, f^{\prime} \in V(F), g, g^{\prime} \in V(G),\left\{f, f^{\prime}\right\} \in E(F) \text { or }\left\{g, g^{\prime}\right\} \in E(G)\right\} .
\end{gathered}
$$

On the other hand, the edge set of the AND-product is given by

$$
\begin{gathered}
E(F \otimes G)=\left\{\left\{(f, g),\left(f^{\prime}, g^{\prime}\right)\right\}: f, f^{\prime} \in V(F), g, g^{\prime} \in V(G),\right. \\
\left.\left\{f, f^{\prime}\right\} \in E(F) \text { and }\left\{g, g^{\prime}\right\} \in E(G) \text {, or } f=f^{\prime},\left\{g, g^{\prime}\right\} \in E(G) \text {, or }\left\{f, f^{\prime}\right\} \in E(F), g=g^{\prime}\right\} .
\end{gathered}
$$

We denote the $t$-fold OR-product of a graph $G$ with itself by $G^{t}$, while the $t$-fold ANDproduct of $G$ with itself will be denoted by $G^{\bigotimes t}$. 
Denoting the complementary graph of a graph $H$ by $\bar{H}$, note that the above definitions imply that $\overline{F \cdot G}=\bar{F} \otimes \bar{G}$. In particular, $\omega\left(G^{t}\right)=\alpha\left(\overline{G^{t}}\right)=\alpha\left(\bar{G}^{\otimes t}\right)$, where $\omega(H)$ and $\alpha(H)$ denote the clique number and the independence number of graph $H$, respectively.

Definition 2. The Shannon OR-capacity of a graph $G$ is defined as the always existing limit

$$
C_{\mathrm{OR}}(G):=\lim _{t \rightarrow \infty} \sqrt[t]{\omega\left(G^{t}\right)}
$$

The Shannon AND-capacity is equal to $C_{\mathrm{AND}}(G):=\lim _{t \rightarrow \infty} \sqrt[t]{\alpha\left(G^{\bowtie t}\right)}=C_{\mathrm{OR}}(\bar{G})$.

We remark, that in information theory Shannon capacity is often defined as the logarithm of the above values (to emphasize its operational meaning), but we will omit logarithms as it is more customarily done in combinatorial treatments. We also note, that all graphs in our discussions are meant to be simple.

The following proposition is quite immediate.

Proposition 3. If $G$ and $H$ are two graphs such that $G \rightarrow H$, then $C_{\mathrm{OR}}(G) \leqslant C_{\mathrm{OR}}(H)$.

Proof. Let $f: V(G) \rightarrow V(H)$ be a graph homomorphism and $\boldsymbol{a}=a_{1} a_{2} \ldots a_{t}, \boldsymbol{b}=$ $b_{1} b_{2} \ldots b_{t}$ be two adjacent vertices of $G^{t}$. Then for some $i$ we have $\left\{a_{i}, b_{i}\right\} \in E(G)$ implying $\left\{f\left(a_{i}\right), f\left(b_{i}\right)\right\} \in E(H)$ and thus $\left\{f\left(a_{1}\right) f\left(a_{2}\right) \ldots f\left(a_{t}\right), f\left(b_{1}\right) f\left(b_{2}\right) \ldots f\left(b_{t}\right)\right\} \in E\left(H^{t}\right)$. Since our graphs are simple (in particular, there are no loops), this implies $\omega\left(G^{t}\right) \leqslant \omega\left(H^{t}\right)$ for every $t$ and thus the statement.

As already said in the Introduction and also appears explicitly in [19] (as Lemma 2.1) once a graph parameter $p$ is nondecreasing via graph homomorphism then it satisfies $p(G \times H) \leqslant \min \{p(G), p(H)\}$ (since $F \times G \rightarrow F$ and $F \times G \rightarrow G$ both hold). Therefore by Proposition 3 we have this inequality for Shannon OR-capacity that we state for future reference.

Corollary 4. $C_{\mathrm{OR}}(F \times G) \leqslant \min \left\{C_{\mathrm{OR}}(F), C_{\mathrm{OR}}(G)\right\}$.

Thus the following question is indeed valid: For what graphs $F$ and $G$ do we have equality in Corollary 4? We elaborate on this question in the next two sections.

\section{On the possibilities of equality}

As already mentioned in the Introduction, if one would be asked whether believing in equality in Corollary 4 sounded plausible, then the most natural reaction seems to be to say "no" based mainly on the fact that the answer to two somewhat similar questions is negative, though neither is trivial. Let us repeat these two questions now also giving the details.

Lovász asked in his celebrated paper [33], whether Shannon OR-capacity is multiplicative with respect to the OR-product. i.e., whether

$$
C_{\mathrm{OR}}(F \cdot G)=C_{\mathrm{OR}}(F) C_{\mathrm{OR}}(G)
$$


holds for all pairs of graphs $F$ and $G$. Note that the left hand side above is always at least as large as the right hand side as a consequence of the easy observation that $\omega\left((F \cdot G)^{k}\right) \geqslant \omega\left(F^{k}\right) \omega\left(G^{k}\right)$ holds for any positive integer $k$. (Formally the question was asked in the complementary language, but its mathematical content was equivalent to this.) This was answered in the negative by Haemers in [22].

The second question is from Shannon's paper [43] and to present it in our language we need the notion of join of two graphs.

Definition 5. The join $F \oplus G$ of graphs $F$ and $G$ has the disjoint union of $V(F)$ and $V(G)$ as vertex set and its edge set is given by

$$
E(F \oplus G)=E(F) \cup E(G) \cup\{\{a, b\}: a \in V(F), b \in V(G)\},
$$

that is, $F \oplus G$ is the disjoint union of graphs $F$ and $G$ with all edges added that has one endpoint in $V(F)$ and the other in $V(G)$.

Shannon [43] proved that $C_{\mathrm{OR}}(F \oplus G) \geqslant C_{\mathrm{OR}}(F)+C_{\mathrm{OR}}(G)$ for all pairs of graphs $F$ and $G$ and formulated the conjecture that equality always holds. This remained open for four decades and was finally refuted by Alon [1].

Two of the graph parameters, the fractional chromatic number $\chi_{f}(G)$ and the Lovász theta number of the complementary graph (or strict vector chromatic number) $\bar{\vartheta}(G)=$ $\vartheta(\bar{G})$ that we mentioned in the Introduction as examples for graph parameters satisfying the Hedetniemi-type equality, i.e., for which we have

$$
\chi_{f}(F \times G)=\min \left\{\chi_{f}(F), \chi_{f}(G)\right\}
$$

and

$$
\bar{\vartheta}(F \times G)=\min \{\bar{\vartheta}(F), \bar{\vartheta}(G)\}
$$

respectively, also satisfy

$$
\chi_{f}(F \cdot G)=\chi_{f}(F) \chi_{f}(G), \quad \bar{\vartheta}(F \cdot G)=\bar{\vartheta}(F) \bar{\vartheta}(G),
$$

and

$$
\chi_{f}(F \oplus G)=\chi_{f}(F)+\chi_{f}(G), \quad \bar{\vartheta}(F \oplus G)=\bar{\vartheta}(F)+\bar{\vartheta}(G) .
$$

As we have also mentioned in the Introduction, both of the above parameters bound Shannon OR-capacity from above, that is, for all graphs $G$ we have

$$
C_{\mathrm{OR}}(G) \leqslant \chi_{f}(G) \text { and } C_{\mathrm{OR}}(G) \leqslant \bar{\vartheta}(G) \text {. }
$$

The first of these two inequalities follows from results in Shannon's paper [43], while the second is due to Lovász [33]. We remark that the second inequality implies the first one as Lovász also proved that $\bar{\vartheta}(G) \leqslant \chi_{f}(G)$ holds for every graph $G$.

The following notion (adapted again for our complementary language) is from Zuiddam's recent paper [60] that borrows the terminology from Strassen's work [47] which it is based on. 
Definition 6. Let $S$ be a collection of graphs closed under the join and the OR-product operations and containing the single vertex graph $K_{1}$. The asymptotic spectrum $Y(S)$ of $S$ is the set of all maps $\varphi: S \rightarrow R_{\geqslant 0}$ which satisfy for all $G, H \in S$ the following four properties:

1. $\varphi\left(K_{1}\right)=1$

2. $\varphi(G \oplus H)=\varphi(G)+\varphi(H)$

3. $\varphi(G \cdot H)=\varphi(G) \cdot \varphi(H)$

4. if $G \rightarrow H$, then $\varphi(G) \leqslant \varphi(H)$.

Note that every $\varphi \in Y(S)$ provides an upper bound for the Shannon OR-capacity of graphs in $S$. Indeed, the first two properties imply $\varphi\left(K_{n}\right)=n$ for every $n$, which together with the fourth property imply $\omega(G) \leqslant \varphi(G)$ for every $G \in S$ and $\varphi \in Y(S)$. Using also the third property we obtain

$$
C_{\mathrm{OR}}(G)=\lim _{t \rightarrow \infty} \sqrt[t]{\omega\left(G^{t}\right)} \leqslant \lim _{t \rightarrow \infty} \sqrt[t]{\varphi\left(G^{t}\right)}=\lim _{t \rightarrow \infty} \sqrt[t]{[\varphi(G)]^{t}}=\varphi(G) .
$$

The two inequalities in (1) are special cases of this fact since both $\chi_{f}(G)$ and $\bar{\vartheta}(G)$ satisfy the conditions in Definition 6 and thus belong to $Y(S)$.

Note also that $C_{\mathrm{OR}}(G)$ itself does not belong to $Y(S)$ by the above mentioned results of Haemers [22] and Alon [1].

Building on Strassen's theory of asymptotic spectra, Zuiddam proved the following surprising result (cf. also [18] for an independently found weaker version).

Theorem 7. (Zuiddam's theorem [60]) Let $S$ be a collection of graphs closed under the join and the OR-product operations and containing the single vertex graph $K_{1}$. Let $Y(S)$ be the asymptotic spectrum of $S$. Then for all graphs $G \in S$ we have

$$
C_{\mathrm{OR}}(G)=\min _{\varphi \in Y(S)} \varphi(G)
$$

That is, Zuiddam's theorem states that the value of $C_{\mathrm{OR}}(G)$ is always equal to the value of one of its "nicely behaving" upper bound functions. Note that this would be trivial if $C_{\mathrm{OR}}(G)$ itself would be a member of $Y(S)$, but we have already seen that this is not the case. Zuiddam [60] gives a list of known elements of $Y(S)$. This list (translated to our complementary language) includes the fractional chromatic number, the Lovász theta number of the complementary graph, the so-called fractional Haemers bound (of the complementary graph) defined in [7] and further investigated in [10, 28], and another parameter called fractional orthogonal rank introduced in [13]. The fractional Haemers' bound also depends on a field and as Zuiddam also remarks, a separation result by Bukh and Cox [10] implies that this family of graph invariants has infinitely many different elements.

Now we are ready to prove our main result. 
Theorem 8. Either

$$
C_{\mathrm{OR}}(F \times G)=\min \left\{C_{\mathrm{OR}}(F), C_{\mathrm{OR}}(G)\right\}
$$

holds for graphs $F$ and $G$ or there exists some function $\varphi$ satisfying the properties given in Definition 6 for which we have

$$
\varphi(F \times G)<\min \{\varphi(F), \varphi(G)\} .
$$

In short, Theorem 8 states that either Shannon OR-capacity satisfies the Hedetniemi-type equality, or if not, then there is some much "nicer behaving" graph invariant, which also violates it.

Proof. Consider two graphs $F$ and $G$ and let $S$ be a class of graphs satisfying the conditions in Zuiddam's theorem and containing all of $F, G$ and $F \times G$. Then by Zuiddam's theorem there exists some $\varphi_{0} \in Y(S)$ for which

$$
C_{\mathrm{OR}}(F \times G)=\varphi_{0}(F \times G) .
$$

Assume that

$$
\varphi_{0}(F \times G)=\min \left\{\varphi_{0}(F), \varphi_{0}(G)\right\}
$$

holds. Then w.l.o.g. we may assume $\varphi_{0}(F) \leqslant \varphi_{0}(G)$ and thus $\varphi_{0}(F \times G)=\varphi_{0}(F)$.

Since all elements in $Y(S)$ are upper bounds on Shannon OR-capacity, we also have that

$$
\min \left\{C_{\mathrm{OR}}(F), C_{\mathrm{OR}}(G)\right\} \leqslant \min \left\{\varphi_{0}(F), \varphi_{0}(G)\right\}=\varphi_{0}(F) .
$$

But

$$
\varphi_{0}(F)=\varphi_{0}(F \times G)=C_{\mathrm{OR}}(F \times G),
$$

so we have obtained

$$
\min \left\{C_{\mathrm{OR}}(F), C_{\mathrm{OR}}(G)\right\} \leqslant C_{\mathrm{OR}}(F \times G) .
$$

Since the opposite inequality is always true (by Corollary 4) this implies

$$
\min \left\{C_{\mathrm{OR}}(F), C_{\mathrm{OR}}(G)\right\}=C_{\mathrm{OR}}(F \times G) .
$$

Consequently, if the last equality does not hold, then we must have $\varphi_{0}(F \times G) \neq$ $\min \left\{\varphi_{0}(F), \varphi_{0}(G)\right\}$ implying

$$
\varphi_{0}(F \times G)<\min \left\{\varphi_{0}(F), \varphi_{0}(G)\right\}=\varphi_{0}(F)
$$

by $\varphi_{0}$ satisfying the fourth property in Definition 6 and the fact that $F \times G \rightarrow F$.

Remark 1. While the proof of Theorem 8 is rather simple it may be worth noting how strong Zuiddam's theorem is which it is based on. An illustration of this is given in the last fifteen minutes of Zuiddam's lecture [61], where he shows, referring to Ron Holzman 
[27], an equally simple proof of the statement (translated to the language and notation we use here), that

$$
C_{\mathrm{OR}}(F \cdot G)=C_{\mathrm{OR}}(F) C_{\mathrm{OR}}(G) \Leftrightarrow C_{\mathrm{OR}}(F \oplus G)=C_{\mathrm{OR}}(F)+C_{\mathrm{OR}}(G)
$$

using his theorem. In other words, in posession of Zuiddam's theorem Haemers' 1979 result [22] about the non-multiplicativity of $C_{\mathrm{OR}}(G)$ with respect to the OR-product already implies Alon's breakthrough result refuting Shannon's conjecture that appeared only almost two decades later. $\diamond$

Remark 2. As already mentioned in the Introduction, graph parameters that satisfy the Hedetniemi-type equality, but violate the conditions in Definition 6 exist. A simple example is the clique number that is not multiplicative with respect to the OR-product. (If it was, then the Shannon-capacity problem would be trivial.) A perhaps more artificial example is the reciprocal of the odd girth (taken to be 0 when the graph is bipartite) which also satisfies the Hedetniemi-type equality but fails to do so with all but the last one of the four conditions in Definition 6. $\diamond$

Remark 3. A more sophisticated such variable as the ones mentioned in Remark 2 is a topological relaxation of the chromatic number in the sense of [45]. There are several closely related lower bounds on the chromatic number based on algebraic topology that all grew out from the pioneering work of Lovász in [32]. In [45] the topological lower bound involving the so-called co-index of the box complex of the graph at hand is considered as a graph parameter itself and it is shown that it also satisfies the Hedetniemi-type equality. (See also [14] and [25] for related results.) This topological lower bound, however, is also not multiplicative under the OR-product. This follows from the fact, that it is a sharp lower bound on the chromatic number of several such graphs (Kneser graphs are a primary example) for which the fractional chromatic number is strictly smaller than the chromatic number. On the other hand it is a well-known theorem by McEliece and Posner [36] (cf. also Berge and Simonovits [6] and see it as Corollary 3.4.3 in [42]) that $\lim _{t \rightarrow \infty} \sqrt[t]{\chi\left(G^{t}\right)}=\chi_{f}(G)$. If the above mentioned lower bound on the chromatic number (that we will simply denote by $\operatorname{top}(G)$ now) was multiplicative under the OR-product, then it would imply by the McEliece-Posner theorem, that $\chi_{f}(G)=\lim _{t \rightarrow \infty} \sqrt[t]{\chi\left(G^{t}\right)} \geqslant$ $\lim _{t \rightarrow \infty} \sqrt[t]{\operatorname{top}\left(G^{t}\right)}=\lim _{t \rightarrow \infty} \sqrt[t]{\operatorname{top}(G)^{t}}=\operatorname{top}(G)$ holds for any graph $G$. In particular, if $\operatorname{top}(G)=\chi(G)$ then we obtained $\chi_{f}(G) \geqslant \chi(G)$, thus by the above mentioned sharpness of $\operatorname{top}(G)$ for, say, Kneser graphs, this would imply that the fractional chromatic number of Kneser graphs equals to their chromatic number, that is far from being true (see more on this in the next remark or in [42]).

Remark 4. Since the appearance of Shitov's paper [44] the primary example for a homomorphism monotone graph parameter that does not satisfy the Hedetniemi-type equality is of course the chromatic number. To see that it does not belong to the asymptotic spectrum one can refer again to the McEliece-Posner theorem [36] mentioned in the previous remark: $\chi(G)$ is not multiplicative under the OR-product, because if it was, then we would have $\chi(G)=\lim _{t \rightarrow \infty} \sqrt[t]{[\chi(G)]^{t}}=\lim _{t \rightarrow \infty} \sqrt[t]{\chi\left(G^{t}\right)}=\chi_{f}(G)$, but there are several graphs $G$ for which $\chi(G)>\chi_{f}(G)$, cf. [42]. 
Another homomorphism monotone graph parameter that does not satisfy the Hedetniemitype equality is provided by the circular chromatic number $\chi_{c}(G)$. As $\chi(G)-1<\chi_{c}(G) \leqslant$ $\chi(G)$ holds for any graph $G$, Shitov's result immediately implies that $\chi_{c}(G)$ does not satisfy the Hedetniemi-type equality either although earlier this was also conjectured to be so by Zhu [55] as a generalization of Hedetniemi's conjecture and it is actually true for graph pairs $(G, H)$ satisfying $\chi_{c}(G \times H)<4$ according to a result of Tardif [48] that generalizes the celebrated theorem of El-Zahar and Sauer [15].

To give a simpler example that does not rely on Shitov's highly nontrivial construction one can consider convex combinations of the chromatic number and the clique number. Define, for example

$$
a(G)=\frac{\omega(G)+\chi(G)}{2} .
$$

It is obvious that $G \rightarrow H$ implies $a(G) \leqslant a(H)$ as a consequence of $\omega(G) \leqslant \omega(H)$ and $\chi(G) \leqslant \chi(H)$. On the other hand if $\omega(H)<\omega(G)$, while $\chi(G)<\chi(H)$, then we have $a(G \times H)<\min \{a(G), a(H)\}$. Such graph pairs are easy to find, the simplest example is perhaps the pair formed by $G=K_{3}$ and $H$ being the 4-chromatic Mycielski graph (also called Grötzsch graph), which is known to be the smallest triangle-free graph with chromatic number 4, see [11]. Indeed, for these two graphs we get $a(G \times H) \leqslant \frac{\chi(G)+\omega(H)}{2}=$ $\frac{5}{2}<3=\min \{a(G), a(H)\}$. (In fact, we have equality in the first inequality as Hedetniemi's conjecture is known to hold when one of the graphs is complete, see e.g. [34], Problem 9.7.c.) An easy way to see that the parameter $a(G)$ does not belong to the asymptotic spectrum, in particular, that it is not multiplicative under the OR-product is the following. Since $\frac{\chi\left(G^{t}\right)}{2} \leqslant \frac{\chi\left(G^{t}\right)+\omega\left(G^{t}\right)}{2} \leqslant \chi\left(G^{t}\right)$ holds, we also have $\lim _{t \rightarrow \infty} \sqrt[t]{a\left(G^{t}\right)}=\lim _{t \rightarrow \infty} \sqrt[t]{\chi\left(G^{t}\right)}=$ $\chi_{f}(G)$, where the last equality is again by the McEliece-Posner theorem. If $a(G)$ was multiplicative under the OR-product, then we would have $\lim _{t \rightarrow \infty} \sqrt[t]{a\left(G^{t}\right)}=a(G)$ and thus $a(G)=\frac{\chi(G)+\omega(G)}{2}=\chi_{f}(G)$. This last equality is false, however, for any graph with $\chi(G)-\chi_{f}(G) \neq \chi_{f}(G)-\omega(G)$. Such graphs are abound, one particular type of example is provided by Kneser graphs $\mathrm{KG}(n, k)$ defined in Definition 12 of the next section for which the fractional chromatic number is $\frac{n}{k}$ (cf. [42]) that one can fix to be, say, 2.1, while their chromatic number $n-2 k+2$ (see Theorem 13) can get arbitrarily large as $n$ grows.

Similar examples can be obtained even by taking linear combinations of two invariants that individually belong to the asymptotic spectrum. Let $\beta, \gamma \in Y(S)$ (we may consider $S$ to be the collection of all finite simple graphs) be two elements of the asymptotic spectrum for which there exists a pair of graphs $G, H \in S$ such that $\beta(G)<\beta(H)$ and $\gamma(H)<\gamma(G)$. Then defining $b(F):=\frac{\beta(F)+\gamma(F)}{2}$ for every graph $F$ we obviously have that $b(F)$ is homomorphism monotone, but $b(G \times H)<\min \{b(G), b(H)\}$. The parameter $b$ is not multiplicative under the OR-product, otherwise we would have $\lim _{t \rightarrow \infty} \sqrt[t]{b\left(F^{t}\right)}=b(F)$ for every graph $F$. However, we must have $\lim _{t \rightarrow \infty} \sqrt[t]{b\left(F^{t}\right)}=\max \{\beta(F), \gamma(F)\}$, which can be equal to $b(F)$ only if $\beta(F)=\gamma(F)$. But our conditions imply that this cannot be the case for every graph: if we have $\beta(G)=\gamma(G)$, then we must have $\gamma(H)<\gamma(G)=$ $\beta(G)<\beta(H)$.

Taking, for example, $\beta=\chi_{f}$ and $\gamma=\bar{\vartheta}$ such a required graph pair exists: it is given, for example, by $G=K_{3}$ and $H$ being the so-called 5-regular Clebsch graph. (The simpler 
term "Clebsch graph" is sometimes used for its complement, which is also called the 10-regular Clebsch graph. The 5-regular Clebsch graph appears, for example, in [21] as a 16 vertex graph whose three isomorphic copies partition the edge set of $K_{16}$ thus establishing the sharp lower bound on the Ramsey number $R(3,3,3)=17$. It is an edge-transitive graph with eigenvalues 5,1 , and -3 , from which the Lovász theta number of its complementary graph can be calculated using results from [33].) Then we have $\chi_{f}(H)=\frac{16}{5}>3$ and $\bar{\vartheta}(H)=\frac{8}{3}<3$, while obviously $\chi_{f}\left(K_{3}\right)=\bar{\vartheta}\left(K_{3}\right)=3$, so the claimed inequalities hold.

We add that several of the so-called multichromatic numbers also do not satisfy the Hedetniemi-type equality, see [49] and [51] for details. $\diamond$

\section{A lower bound and identifying test cases}

Due to the lack of knowledge of the Shannon capacity value for many graphs (as already mentioned in the Introduction even that is not known whether the computational problem given by it is decidable, see [2]), it is not entirely trivial how to find a pair of graphs on which one could at least try checking whether there is equality in Corollary 4 in any nontrivial way. In this section we establish a general lower bound for $C_{\mathrm{OR}}(F \times G)$ and present some graph pairs for which this lower bound is strictly smaller than the upper bound $\min \left\{C_{\mathrm{OR}}(F), C_{\mathrm{OR}}(G)\right\}$. Whether either of the two bounds is sharp in these cases remains an open problem.

\subsection{Lower bound}

For two graphs $X$ and $Y$ we denote by $X \subseteq Y$ if $X$ is a (not necessarily induced) subgraph of $Y$. The following proposition gives our lower bound.

\section{Proposition 9.}

$$
C_{\mathrm{OR}}(F \times G) \geqslant \max \left\{C_{\mathrm{OR}}\left(F^{\prime}\right), C_{\mathrm{OR}}\left(G^{\prime}\right): F^{\prime} \subseteq F, F^{\prime} \rightarrow G, G^{\prime} \subseteq G, G^{\prime} \rightarrow F\right\} .
$$

Proof. Let $F_{0}$ denote the subgraph $F^{\prime}$ of $F$ that admits a homomorphism to $G$ with largest value of $C_{\mathrm{OR}}\left(F^{\prime}\right)$. Let $G_{0}$ be the analogous subgraph of $G$ obtained when we exchange the letters $F$ and $G$ in the previous sentence. The statement is equivalent to the inequality $C_{\mathrm{OR}}(F \times G) \geqslant \max \left\{C_{\mathrm{OR}}\left(F_{0}\right), C_{\mathrm{OR}}\left(G_{0}\right)\right\}$.

Thus it is enough to show $C_{\mathrm{OR}}(F \times G) \geqslant C_{\mathrm{OR}}\left(F_{0}\right)$, the same argument will prove $C_{\mathrm{OR}}(F \times G) \geqslant C_{\mathrm{OR}}\left(G_{0}\right)$ when exchanging the role of $F$ and $G$.

This readily follows from Proposition 3 and the well-known fact (see for example [26], Proposition 2.1 on page 37), that $X \rightarrow F$ and $X \rightarrow G$ implies $X \rightarrow F \times G$. Putting $F_{0}$ in the role of $X$ here, we have $F_{0} \rightarrow F$ and $F_{0} \rightarrow G$ by the assumptions and thus $C_{O R}\left(F_{0}\right) \leqslant C_{\mathrm{OR}}(F \times G)$ follows by $F_{0} \rightarrow F \times G$.

Corollary 10. If $F \rightarrow G$ then $C_{\mathrm{OR}}(F \times G)=C_{\mathrm{OR}}(F)$.

Proof. This is an immediate consequence of Proposition 9 and Corollary 4. 
Naturally, if we would like to "test" whether the inequality in Corollary 4 can be strict then we need a pair of graphs $F$ and $G$ for which the upper bound on $C_{\mathrm{OR}}(F \times G)$ provided by Corollary 4 is strictly larger than the lower bound given in Proposition 9 . As the exact value of Shannon capacity is known only in a few nontrivial cases, finding such a pair is not a completely trivial matter. We discuss this problem in the following two subsections.

To make it easier to refer to the lower and upper bounds proven in Proposition 9 and Corollary 4, respectively, we introduce for them the following notations.

Definition 11. For two graphs $F$ and $G$ we define

$$
L B(F, G):=\max \left\{C_{\mathrm{OR}}\left(F^{\prime}\right), C_{\mathrm{OR}}\left(G^{\prime}\right): F^{\prime} \subseteq F, F^{\prime} \rightarrow G, G^{\prime} \subseteq G, G^{\prime} \rightarrow F\right\}
$$

and

$$
U B(F, G):=\min \left\{C_{\mathrm{OR}}(F), C_{\mathrm{OR}}(G)\right\} .
$$

A pair of graphs $(F, G)$ will be called a trivial pair if

$$
L B(F, G)=U B(F, G)
$$

Correspondingly, a nontrivial pair will refer to a pair of graphs $(F, G)$ for which

$$
L B(F, G)<U B(F, G)
$$

\subsection{Trivial pairs}

Although determining the value of Shannon capacity is a notoriously hard problem in general, there are several cases when it is almost trivial. Most notably, it is well-known that $\omega(G) \leqslant C_{\mathrm{OR}}(G) \leqslant \chi(G)$ holds for all graphs $G$, thus whenever $\chi(G)=\omega(G)$, then we automatically have $C_{\mathrm{OR}}(G)=\omega(G)$. In particular, this holds for all perfect graphs, whose definition was actually inspired by the above fact, cf. [5]. There are other cases when $C_{\mathrm{OR}}(G)=\omega(G)$ holds for less trivial reasons. First of all, as already mentioned, the fractional chromatic number $\chi_{f}(G)$ also bounds Shannon OR-capacity from above, thus already $\omega(G)=\chi_{f}(G)$ implies $C_{\mathrm{OR}}(G)=\omega(G)$. Since $\chi_{f}(G) \leqslant \chi(G)$ holds for all graphs $G$ (see [42] for more details), the criterion $\omega(G)=\chi_{f}(G)$ is less restrictive than $\omega(G)=\chi(G)$. Famous examples of graphs for which $\omega(G)=\chi_{f}(G)<\chi(G)$ are provided by certain Kneser graphs.

Definition 12. Let $n, k$ be positive integers satisfying $n \geqslant 2 k$. The Kneser graph $\mathrm{KG}(n, k)$ is defined as

$$
V(\operatorname{KG}(n, k))=\left(\begin{array}{c}
{[n]} \\
k
\end{array}\right),
$$

where $[n]=\{1,2, \ldots, n\}$ and $\left(\begin{array}{c}{[n]} \\ k\end{array}\right)$ stands for all $k$-element subsets of $[n]$, while

$$
E(\operatorname{KG}(n, k))=\left\{A B: A, B \in\left(\begin{array}{c}
{[n]} \\
k
\end{array}\right), A \cap B=\varnothing\right\}
$$


It is trivial from the definition that $\omega(\operatorname{KG}(n, k))=\left\lfloor\frac{n}{k}\right\rfloor$, while it is well-known (see e.g. [42]) that $\chi_{f}(\operatorname{KG}(n, k))=\frac{n}{k}$ holds for all $n \geqslant 2 k$. We also mention that

$$
\alpha(\operatorname{KG}(n, k))=\left(\begin{array}{l}
n-1 \\
k-1
\end{array}\right)
$$

follows from the famous Erdös-Ko-Rado theorem [16] and quote the following celebrated result of Lovász verifying a conjecture of Kneser [31] which implies $\chi_{f}(\operatorname{KG}(n, k))<$ $\chi(\mathrm{KG}(n, k))$ whenever $k>1$ and $n / k>2$. (For $k=1 \mathrm{KG}(n, k)=K_{n}$ and for $n / k=2$ $\mathrm{KG}(n, k)$ is bipartite, that is, in these cases the Kneser graph is perfect.)

Theorem 13. (Lovász-Kneser theorem [32]) For all $n \geqslant 2 k$

$$
\chi(\operatorname{KG}(n, k))=n-2 k+2 .
$$

Thus we have $C_{\mathrm{OR}}(\mathrm{KG}(n, k))=\omega(\operatorname{KG}(n, k))<\chi(\operatorname{KG}(n, k))$ whenever $k>1$ and $2<n / k$ is integral.

An even more general case when $C_{\mathrm{OR}}(G)=\omega(G)$ is provided by the condition $\bar{\vartheta}(G)=$ $\omega(G)$ since in general the chain of inequalities

$$
\omega(G) \leqslant C_{\mathrm{OR}}(G) \leqslant \bar{\vartheta}(G) \leqslant \chi_{f}(G)
$$

holds. In [33] Lovász proved the following generalization of the Erdős-Ko-Rado theorem.

Theorem 14. (Lovász [33]) For all $n \geqslant 2 k$ we have

$$
\bar{\vartheta} \overline{(\mathrm{KG}(n, k))}=\left(\begin{array}{c}
n-1 \\
k-1
\end{array}\right)=\omega(\overline{\mathrm{KG}(n, k)})
$$

for the complementary graph of the Kneser graph $\mathrm{KG}(n, k)$, where the last equality is simply the Erdös-Ko-Rado theorem.

This implies that the Shannon OR-capacity of the complementary graph of the Kneser graph also equals its clique number. Consequences of the foregoing can be summarized in the following statement.

Proposition 15. The graph pair $(A, B)$ forms a trivial pair whenever

$$
C_{\mathrm{OR}}(A)=\omega(A) \text { and } C_{\mathrm{OR}}(B)=\omega(B)
$$

both hold. This happens in particular, when both of the graphs $A$ and $B$ are one of the following types: a perfect graph, a Kneser graph $\mathrm{KG}(n, k)$ where $k$ divides $n$, or the complementary graph of a Kneser graph.

Proof. Let $(A, B)$ be a graph pair satisfying the condition and without loss of generality assume, that $\omega(A) \leqslant \omega(B)$. Then we have $K_{\omega(A)} \rightarrow B$ and therefore $L B(A, B) \geqslant$ $C_{\mathrm{OR}}\left(K_{\omega(A)}\right)=\omega(A)$. On the other hand,

$$
U B(A, B)=\min \left\{C_{\mathrm{OR}}(A), C_{\mathrm{OR}}(B)\right\}=\min \{\omega(A), \omega(B)\}=\omega(A)
$$


holds by the conditions. Thus

$$
\omega(A) \leqslant L B(A, B) \leqslant U B(A, B)=\omega(A),
$$

so we must have equality.

The second part of the statement claiming that the types of graphs listed satisfy the condition follows from the discussion preceding the proposition.

Remark 5. The proof of Proposition 15 shows that it is enough to have $C_{\mathrm{OR}}(A)=$ $\omega(A) \leqslant \omega(B)$ for the conclusion. That is $(A, B)$ is a trivial pair whenever the Shannon OR-capacity of that one of them which has the smaller clique number is equal to this clique number, we do not need the similar equality for the other graph. I stated the proposition in the above less general form, because this way it sounds perhaps less technical. $\diamond$

Proposition 15 lists graph pairs that form trivial pairs and this is known because we know the value of their Shannon OR-capacity. It can also happen that we do not really know the actual value of the Shannon OR-capacities of the graphs involved, yet by Corollary 10 we know the equality of our two bounds.

The primary example for a graph with larger Shannon OR-capacity than clique number is the cycle of length five $C_{5}$ for which we know $C_{\mathrm{OR}}\left(C_{5}\right)=\sqrt{5}>2=\omega\left(C_{5}\right)$ by Shannon [43] and Lovász [33]. Shannon's observation about $C_{\mathrm{OR}}\left(C_{5}\right)>\omega\left(C_{5}\right)$ was generalized by Bohman and Holzman [9], who proved that for any odd cycle $C_{2 k+1}$ one has $C_{\mathrm{OR}}\left(C_{2 k+1}\right)>$ 2 , therefore for any odd cycle of length at least 5 , the value of its Shannon OR-capacity is strictly larger than its clique number. (The actual value of $C_{\mathrm{OR}}\left(C_{2 k+1}\right)$ is famously unknown for any $k>2$, that is for any odd cycle of length at least 7.) Since a longer odd cycle always admits a homomorphism to a shorter one (but not vice versa) for arbitrary integers $1 \leqslant k \leqslant \ell$ we have $C_{\mathrm{OR}}\left(C_{2 k+1} \times C_{2 \ell+1}\right)=C_{\mathrm{OR}}\left(C_{2 \ell+1}\right)$. In particular, we can state the following consequence of Corollary 10.

Claim 16. The graph pair $\left(C_{2 k+1}, C_{2 \ell+1}\right)$ is a trivial pair for any two positive integers $k$ and $\ell$.

Other types of trivial pairs can be given by taking any graph $G$ of possibly unknown Shannon OR-capacity value and known chromatic number $\chi(G)$ and considering the graph pair $\left(G, K_{\chi(G)}\right)$. Obviously, this is a trivial pair by Corollary 10. In particular, $\left(\mathrm{KG}(n, k), K_{n-2 k+2}\right)$ is such a pair by the Lovász-Kneser theorem with

$$
L B\left(\operatorname{KG}(n, k), K_{n-2 k+2}\right)=U B\left(\operatorname{KG}(n, k), K_{n-2 k+2}\right)=C_{\mathrm{OR}}(\mathrm{KG}(n, k)),
$$

and we know this in spite of not knowing the exact value of the right hand side unless $k$ is a divisor of $n$. (How little is known about $C_{\mathrm{OR}}(\mathrm{KG}(n, k))=C_{\mathrm{AND}}(\overline{\mathrm{KG}(n, k)})$ when $k$ does not divide $n$ is indicated by the discussion in the paper [30].) If we change the complete graph in this pair to a slightly smaller one, for example, we consider the pair $\left(\mathrm{KG}(n, k), K_{n-2 k+1}\right)$ then we get a pair that may easily be a nontrivial pair but proving this seems to be problematic. We may assume that $n / k \leqslant n-2 k+1$ and thus

$$
U B\left(\mathrm{KG}(n, k), K_{n-2 k+1}\right)=\min \left\{C_{\mathrm{OR}}(\mathrm{KG}(n, k)), C_{\mathrm{OR}}\left(K_{n-2 k+1}\right)=\right.
$$




$$
\min \left\{C_{\mathrm{OR}}(\mathrm{KG}(n, k)), n-2 k+1\right\}=C_{\mathrm{OR}}(\mathrm{KG}(n, k))
$$

since $C_{\mathrm{OR}}(\mathrm{KG}(n, k)) \leqslant \chi_{f}(\mathrm{KG}(n, k))=n / k \leqslant n-2 k+1$, but it is still problematic to decide how this $C_{\mathrm{OR}}(\mathrm{KG}(n, k))$ value relates to $L B\left(\mathrm{KG}(n, k), K_{n-2 k+1}\right)$ that will be the largest Shannon OR-capacity attained by an $(n-2 k+1)$-chromatic subgraph of the Kneser graph. This is mentioned only to emphasize the difficulty of coming up with a graph pair for which its nontriviality can be proven, the problem we consider in the next subsection.

\subsection{Nontrivial pairs}

The nontriviality of graph pairs we present in this subsection will be based on properties of Paley graphs and their variants.

Definition 17. Let $q$ be an odd prime power satisfying $q \equiv 1(\bmod 4)$. The Paley graph $P_{q}$ is defined on the elements of the finite field $F_{q}$ as vertices. Two vertices form an edge if and only if their difference in $F_{q}$ is a square in $F_{q}$.

Note that the condition on $q$ ensures that -1 has a square root in $F_{q}$ and thus $a-b$ is a square in $F_{q}$ if and only if $b-a$ is. Thus the definition is indeed meaningful and results in a(n undirected) graph. In the special case when $q$ itself is a prime number $p$, edges of $P_{p}$ are between vertices whose difference is a quadratic residue modulo $p$.

We also remark that $P_{5}$ is just the five-cycle $C_{5}$ and the graph $P_{17}$ is well-known to be the unique graph on 17 vertices not having either a clique or an independent set of size 4 , thus establishing the sharp lower bound on the largest known diagonal Ramsey number $R(4,4)=18$, see [21]. (In fact, $P_{5} \cong C_{5}$ is the unique graph establishing $R(3,3) \geqslant 6$. For more on the connection between Ramsey numbers and Shannon capacity, cf. [3, 17, 37].)

Paley graphs are well-known to be self-complementary, vertex-transitive, and also edgetransitive, cf. e.g. [54, 39]. The first two of these properties make them particularly useful for us by the following theorem of Lovász.

Theorem 18. (Lovász [33]) If $G$ is a vertex-transitive self-complementary graph on $n$ vertices, then

$$
C_{\mathrm{OR}}(G)=\sqrt{n}
$$

Thus we have $C_{\mathrm{OR}}\left(P_{q}\right)=\sqrt{q}$ for all prime powers $q \equiv 1(\bmod 4)$.

Let $p \equiv 1(\bmod 4)$ be a prime number. The value of the clique number (or equivalently, the independence number) of $P_{p}$ is not known and determining it is a well-known unsolved problem in number theory, the conjectured value being $O\left((\log p)^{2}\right)$, cf. [35]. It is not hard to see (and also follows from Theorem 18 above) that $\omega\left(P_{p}\right) \leqslant \sqrt{p}$. Improving this bound just by 1 for infinitely many primes $p$ was already a nontrivial task that was achieved by 
Bachoc, Matolcsi, and Ruzsa [4] only a few years ago. Recently Hanson and Petridis [23] managed to improve this substantially by proving the general upper bound

$$
\omega\left(P_{p}\right) \leqslant \frac{\sqrt{2 p-1}+1}{2}<\frac{\sqrt{2 p}+1}{2} .
$$

Notice that this upper bound immediately implies

$$
\chi\left(P_{p}\right)>\frac{2 p}{\sqrt{2 p}+1}>\sqrt{2 p}-1
$$

by $\chi\left(P_{p}\right) \geqslant \frac{\left|V\left(P_{p}\right)\right|}{\alpha\left(P_{p}\right)}=\frac{\left|V\left(P_{p}\right)\right|}{\omega\left(P_{p}\right)}$. This in turn gives $\chi\left(P_{p}\right)>\sqrt{p}+1 \geqslant\lceil\sqrt{p}\rceil$, whenever $\sqrt{2 p}-1>\sqrt{p}+1$ that is, $\sqrt{p}>\frac{2}{\sqrt{2}-1}$, which is true whenever $p \geqslant 23$. This means that for primes at least 23 the largest subgraph of $P_{p}$ that can be colored with $\lceil\sqrt{p}\rceil$ colors has strictly fewer vertices than $P_{p}$ itself. There are only three primes of the form $4 k+1$ below 23: $5,13,17$. As already mentioned above, $P_{17}$ is well-known from [21] to be the graph establishing the largest known diagonal Ramsey number $R(4,4)=18$, that is, it has no clique or independent set on more than 3 vertices. In fact, this also follows from the Hanson-Petridis bound as well as $\alpha\left(P_{13}\right) \leqslant 3$. Therefore we have $\chi\left(P_{17}\right) \geqslant\left\lceil\frac{\left|V\left(P_{17}\right)\right|}{\alpha\left(P_{17}\right)}\right\rceil \geqslant$ $\left\lceil\frac{17}{3}\right\rceil=6>\lceil\sqrt{17}\rceil=5$ as well as $\chi\left(P_{13}\right) \geqslant\left\lceil\frac{13}{3}\right\rceil=5>\lceil\sqrt{13}\rceil=4$. (Obviously, the analogous strict inequality does not hold for $P_{5} \cong C_{5}$.) We summarize these facts as a consequence of the Hanson-Petridis upper bound.

Corollary HP. If $p>5$ is a prime number satisfying $p \equiv 1(\bmod 4)$, then

$$
\chi\left(P_{p}\right)>\lceil\sqrt{p}\rceil .
$$

In particular, the largest subgraph of $P_{p}$ that can be colored with $\lceil\sqrt{p}\rceil$ colors has fewer than $p$ vertices.

Our goal is to present graph pairs $(A, B)$ for which the lower bound $L B(A, B)$ of Proposition 9 is strictly smaller than the upper bound $U B(A, B)=\min \left\{C_{\mathrm{OR}}(A), C_{\mathrm{OR}}(B)\right\}$ given by Corollary 4 . The following conjecture, if true, gives infinitely many such pairs. A few special cases we are going to verify using the computability of the Lovász theta number and a program available for its calculation. Afterwards we also give some other graph pairs that are candidates for the above strict inequality and are variants of those in the conjecture.

Conjecture 19. Let $p>5$ be a prime number satisfying $p \equiv 1(\bmod 4)$ and set $m:=$ $\lceil\sqrt{p}\rceil$. Then the graph pair consisting of the Paley graph $P_{p}$ and the complete graph $K_{m}$ has the property

$$
L B\left(P_{p}, K_{m}\right)<\sqrt{p}=U B\left(P_{p}, K_{m}\right) .
$$

Note that the equality $U B\left(P_{p}, K_{m}\right)=\sqrt{p}$ above is a fact that follows from Lovász's Theorem 18 quoted above, the trivial equality $C_{\mathrm{OR}}\left(K_{m}\right)=m$ and our choice of $m=\lceil\sqrt{p}\rceil$. Thus the conjecture concerns $L B\left(P_{p}, K_{m}\right)$ being strictly less than $\sqrt{p}$. To present our argument supporting this conjecture and its variants we introduce some more notation. 
Definition 20. For a prime number $p$ satisfying $p \equiv 1(\bmod 4)$ let $Q_{p-1}$ be the graph obtained by deleting a vertex of the Paley graph $P_{p}$. Note that since $P_{p}$ is vertex-transitive (the isomorphy class of) $Q_{p-1}$ does not depend on which vertex is deleted.

Furthermore, let $Z_{p-2}^{(a)}$ denote the graph obtained by deleting two adjacent vertices of $P_{p}$, while $Z_{p-2}^{(n)}$ denote the graph obtained by deleting two nonadjacent vertices of $P_{p}$. As the Paley graph $P_{p}$ is edge-transitive and self-complementary, (the isomorphy class of) neither $Z_{p-2}^{(a)}$ nor $Z_{p-2}^{(n)}$ depends on which two adjacent or nonadjacent vertices are deleted.

Below we state another conjecture and then show that it would imply Conjecture 19.

Conjecture 21. For every prime number $p$ satisfying $p \equiv 1(\bmod 4)$

$$
C_{\mathrm{OR}}\left(Q_{p-1}\right)<C_{\mathrm{OR}}\left(P_{p}\right)
$$

Proposition 22. Conjecture 21 implies Conjecture 19.

Proof. Let $p>5$ be a prime number satisfying $p \equiv 1(\bmod 4)$ and set $m:=\lceil\sqrt{p}\rceil$. We already noted (right after stating Conjecture 19) that we have $U B\left(P_{p}, K_{m}\right)=\sqrt{p}$ under these assumptions, thus Conjecture 19 essentially means $L B\left(P_{p}, K_{m}\right)<\sqrt{p}$.

The upper bound of Hanson and Petridis on $\omega\left(P_{p}\right)$ implies that $K_{m}$ does not admit a homomorphism to $P_{p}$, therefore

$$
\max \left\{C_{\mathrm{OR}}(H): H \subseteq K_{m}, H \rightarrow P_{p}\right\} \leqslant m-1<\sqrt{p} .
$$

On the other hand, we know from Corollary HP that any subgraph $Q$ of $P_{p}$ admitting a homomorphism to $K_{m}$ has strictly fewer vertices than $P_{p}$ itself and thus must be a subgraph of $Q_{p-1}$, too. Thus by the monotonicity of Shannon OR-capacity, we must have $C_{\mathrm{OR}}(Q) \leqslant C_{\mathrm{OR}}\left(Q_{p-1}\right)$. Assuming that Conjecture 21 is true, this implies

$$
\max \left\{C_{\mathrm{OR}}(Q): Q \subseteq P_{p}, Q \rightarrow K_{m}\right\} \leqslant C_{\mathrm{OR}}\left(Q_{p-1}\right)<C_{\mathrm{OR}}\left(P_{p}\right)=\sqrt{p} .
$$

The inequalities (2) and (3) imply $L B\left(P_{p}, K_{m}\right)<\sqrt{p}$ as needed.

Remark 6. It feels unfortunate that we do not have a proof of Conjecture 21. Though we believe it is true for any prime $p$ (of the form $4 k+1$ ) it is clear that this will not follow just from the symmetry properties of $P_{p}$ (that one might believe at first sight), as the analogous statement is not true for all prime powers $q$. Indeed, if $q=p^{k}$ for $k$ even, then it is known that $\omega\left(P_{q}\right)=\sqrt{q}$, see [8] (cf. also [4]), and by vertex-transitivity this immediately implies $\omega\left(Q_{q-1}\right)=\sqrt{q}$ as well, that further implies $C_{\mathrm{OR}}\left(Q_{q-1}\right)=\sqrt{q}$ (where analogously to $Q_{p-1}, Q_{q-1}$ denotes the graph obtained by deleting a vertex of $\left.P_{q}\right)$. $\diamond$

Although we do not have a general proof for $C_{\mathrm{OR}}\left(Q_{p-1}\right)<C_{\mathrm{OR}}\left(P_{p}\right)$, using the computability of the upper bound provided by the Lovász theta number on Shannon OR-capacity we can decide in several cases that this indeed happens. With the help of the online available 
Python code [46] to compute the Lovász theta number for specific graphs ${ }^{1}$, we can obtain for example the following values for the first five relevant numbers:

$$
\begin{gathered}
\bar{\vartheta}\left(Q_{12}\right) \approx 3.4927<\sqrt{13}, \quad \bar{\vartheta}\left(Q_{16}\right) \approx 4.0035<\sqrt{17} \\
\bar{\vartheta}\left(Q_{28}\right) \approx 5.3069<\sqrt{29}, \quad \bar{\vartheta}\left(Q_{36}\right) \approx 6.0025<\sqrt{37}, \quad \bar{\vartheta}\left(Q_{40}\right) \approx 6.3493<\sqrt{41} .
\end{gathered}
$$

In the light of Proposition 22 this leads to the following.

Claim 23. The pairs of graphs $\left(P_{13}, K_{4}\right),\left(P_{17}, K_{5}\right),\left(P_{29}, K_{6}\right),\left(P_{37}, K_{7}\right),\left(P_{41}, K_{7}\right)$ satisfy Conjecture 19, that is they form nontrivial pairs.

Further examples of graph pairs for which our lower and upper bounds do not coincide can be obtained by using the graphs $Q_{p-1}$ in place of the Payley graphs $P_{p}$. What makes these examples interesting is the fact that $p$ can be chosen so in several cases that our lower bound $L B(A, B)$ obtained when considering $A=Q_{p-1}$ and $B$ an appropriately chosen complete graph as our graph pair $(A, B)$ is an integer. (Although the relevance of this is questionable, one might believe that it may be easier to decide whether an integral lower bound is sharp or not.)

Let us start this part of our discussion by stating the following well-known and easy fact.

Fact 24. Let $p$ be a prime number satisfying $p \equiv 1(\bmod 4)$. Then the graph $Q_{p-1}$ is self-complementary and therefore

$$
C_{\mathrm{OR}}\left(Q_{p-1}\right) \geqslant \sqrt{p-1}
$$

The above claimed self-complementarity of $Q_{p-1}$ is a well-known consequence of the structural description of self-complementary graphs [40]. (It follows easily from the observation that each cycle in the cycle decomposition of a complementing permutation of the vertices of a self-complementary graph should have even length with the possible exception of one cycle of length 1 that necessarily appears when the number of vertices is odd.) The second part of the above Fact is also easy and well-known: if $G$ is a self-complementary graph on vertex set $[n]=\{1, \ldots, n\}$ and $\sigma$ is a complementing permutation of $[n]$, then the set of $n$ sequences of length 2 given by $\{(i, \sigma(i)): i \in[n]\}$ forms a clique of size $n$ in the second OR-power of $G$ implying $C_{\mathrm{OR}}(G) \geqslant \sqrt{n}$.

The main difference in using $C_{\mathrm{OR}}\left(Q_{p-1}\right) \geqslant \sqrt{p-1}$ compared to $C_{\mathrm{OR}}\left(P_{p}\right)=\sqrt{p}$ is that if $p$ is a prime number then $\sqrt{p}$ can never be integral, but $\sqrt{p-1}$ can be. This happens in several cases, starting (disregarding $p=2,5$ that are not relevant for us) with $p=$ 17,37, 101 (cf. sequence A002496 of The Online Encyclopedia of Integer Sequences [38]). Whether this sequence (that is the sequence of primes that are 1 larger than the square of an integer) is infinite (as it is believed to be) is a famous open problem in number theory

\footnotetext{
${ }^{1}$ I am grateful to Anna Gujgiczer for showing me how this code can be used and also for providing several of the required calculations.
} 
(one of the four problems called Landau's problems along with Goldbach's conjecture, the twin-prime conjecture, and Legendre's conjecture).

Based on the above remarks the following conjecture, parallel to Conjecture 19, can be formulated.

Conjecture 25. Let $p>5$ be a prime number for which $p-1=s^{2}$ for some positive integer $s$. Then

$$
L B\left(Q_{p-1}, K_{s}\right)<s=U B\left(Q_{p-1}, K_{s}\right) .
$$

Here again, $s=\min \left\{C_{\mathrm{OR}}\left(Q_{p-1}\right), C_{\mathrm{OR}}\left(K_{s}\right)\right\}$ is a fact that follows from our discussion above, so the real content of the conjecture is that $L B\left(Q_{p-1}, K_{s}\right)<s$ holds. This would follow from the following conjecture that is the analog of Conjecture 21 in this setting.

Conjecture 26. For every prime number $p>5$ satisfying $p=s^{2}+1$ for some positive integer $s$

$$
\max \left\{C_{\mathrm{OR}}\left(Z_{p-2}^{(a)}\right), C_{\mathrm{OR}}\left(Z_{p-2}^{(n)}\right)\right\}<s .
$$

Proposition 27. Conjecture 26 implies Conjecture 25.

Proof. The upper bound of Hanson and Petridis on $\omega\left(P_{p}\right)$ implies that $K_{s}$ does not admit a homomorphism to $P_{p}$, therefore it also does not admit a homomorphism into $Q_{p-1} \subseteq P_{p}$. (In fact, vertex-transitivity of $P_{p}$ implies that $\omega\left(Q_{p-1}\right)=\omega\left(P_{p}\right)$.) Thus we have

$$
\max \left\{C_{\mathrm{OR}}(H): H \subseteq K_{s}, H \rightarrow Q_{p-1}\right\} \leqslant s-1 .
$$

On the other hand, the Hanson-Petridis upper bound on $\omega\left(P_{p}\right)=\alpha\left(P_{p}\right)=\alpha\left(Q_{p-1}\right)$ implies that $\chi\left(Q_{p-1}\right) \geqslant\left\lceil\frac{p-1}{\alpha\left(Q_{p-1}\right)}\right]>s$ whenever $s \geqslant 4$ (which is always the case for us as $p>5$ ). Therefore any subgraph $Z$ of $Q_{p-1}$ admitting a homomorphism to $K_{s}$ has strictly fewer vertices than $p-1$ and thus must be a subgraph of at least one of $Z_{p-2}^{(a)}$ and $Z_{p-2}^{(n)}$. By the monotonicity of Shannon OR-capacity this implies that $C_{\mathrm{OR}}(Z) \leqslant$ $\max \left\{C_{\mathrm{OR}}\left(Z_{p-2}^{(a)}\right), C_{\mathrm{OR}}\left(Z_{p-2}^{(n)}\right)\right\}$. Assuming the validity of Conjecture 26 this implies

$$
\max \left\{C_{\mathrm{OR}}(Z): Z \subseteq Q_{p-1}, Z \rightarrow K_{s}\right\} \leqslant \max \left\{C_{\mathrm{OR}}\left(Z_{p-2}^{(a)}\right), C_{\mathrm{OR}}\left(Z_{p-2}^{(n)}\right)\right\}<s .
$$

The inequalities (4) and (5) imply $L B\left(Q_{p-1}, K_{s}\right)<s$ as needed.

As it is the case with Conjecture 21 we also do not have a proof of Conjecture 26. But using again the Python code [46] we can obtain some values that support its validity and provide us some particular pairs of graphs for which our two bounds differ.

In particular, we obtain that

$$
\begin{aligned}
& \max \left\{\bar{\vartheta}\left(Z_{15}^{(a)}\right), \bar{\vartheta}\left(Z_{15}^{(n)}\right)\right\}=\max \{3.8726,3.8849\}<4, \\
& \max \left\{\bar{\vartheta}\left(Z_{35}^{(a)}\right), \bar{\vartheta}\left(Z_{35}^{(n)}\right)\right\}=\max \{5.9128,5.9251\}<6,
\end{aligned}
$$

and

$$
\max \left\{\bar{\vartheta}\left(Z_{99}^{(a)}\right), \bar{\vartheta}\left(Z_{99}^{(n)}\right)\right\}=\max \{9.9496,9.9574\}<10 .
$$

Thus we have the following. 
Claim 28. Each of the graph pairs $\left(Q_{16}, K_{4}\right),\left(Q_{36}, K_{6}\right)$, and $\left(Q_{100}, K_{10}\right)$ satisfy Conjecture 25, that is they form nontrivial pairs.

Remark 7. Concerning the pair $\left(Q_{16}, K_{4}\right)$ it is perhaps interesting to remark that while $L B\left(Q_{16}, K_{4}\right)<4$ we also have $L B\left(Q_{16}, K_{4}\right) \geqslant 1+\sqrt{5}>3$. This follows from the fact that $P_{17}$ and thus $Q_{16}$, too, contains as a subgraph the 6-vertex wheel graph $W_{6}=C_{5} \oplus K_{1}$, whose Shannon OR-capacity is known to be $1+C_{\mathrm{OR}}\left(C_{5}\right)=1+\sqrt{5}$, cf. [43, 1$]$.

Thus the graph pairs listed in Claims 23 and 28 provide "test cases" for investigating the possibility of equality in Corollary 4 . Let us stress again, that in the light of Theorem 8 any proof showing for example $C_{\mathrm{OR}}\left(Q_{16} \times K_{4}\right)<4$ would imply the existence of a graph parameter that satisfies all the four conditions in Definition 6 and yet fails to satisfy the Hedetniemi-type equality.

\section{Acknowledgements}

Many thanks are due to Anna Gujgiczer for calculating the values of $\bar{\vartheta}$ for some relevant subgraphs of Paley graphs with the help of Stahlke's code [46] and for explaining me how this code can be used. I also thank Kati Friedl for a useful discussion.

\section{References}

[1] Noga Alon, The Shannon capacity of a union, Combinatorica, 18 (1998), 301-310.

[2] Noga Alon, Graph powers, in: Contemporary combinatorics, Bolyai Soc. Math. Stud., 10, János Bolyai Math. Soc., Budapest, 2002, 11-28.

[3] Noga Alon, Alon Orlitsky, Repeated communication and Ramsey graphs, IEEE Trans. Inform. Theory, 41 (1995), 1276-1289.

[4] Christine Bachoc, Máté Matolcsi, Imre Z. Ruzsa, Squares and difference sets in finite fields, Integers, 13 (2013), Paper No. A77, 5 pp.

[5] Claude Berge, Motivations and history of some of my conjectures, Discrete Math., 165/166 (1997), 61-70.

[6] Claude Berge, Miklós Simonovits, The coloring numbers of the direct product of two hypergraphs, in: Hypergraph Seminar (C. Berge and D. Ray-Chaudhury, eds.), Lecture Notes in Mathematics 411, Springer-Verlag, 1974, 21-33.

[7] Anna Blasiak, A graph-theoretic approach to network coding, PhD thesis, Cornell University, 2013.

[8] Aart Blokhuis, On subsets of $G F\left(q^{2}\right)$ with square differences, Indag. Math., 87 (4) (1984), 369-372.

[9] Tom Bohman, Ron Holzman, A nontrivial lower bound on the Shannon capacity of the complements of odd cycles, IEEE Trans. Inform. Theory, 49 (2003), 721-722.

[10] Boris Bukh, Christopher Cox, On a fractional version of Haemers' bound, IEEE Trans. Inform. Theory, 65 (2019), no. 6, 3340-3348; arXiv:1802.00476. 
[11] Václav Chvátal, The minimality of the Mycielski graph, in: Graphs and Combinatorics (R. A. Bari and F. Harary eds.), Lecture Notes in Mathematics, 406., Springer, Berlin, Heidelberg, 1974.

[12] Imre Csiszár, János Körner, Information Theory. Coding Theorems for Discrete Memoryless Systems. Second Edition., Cambridge University Press, Cambridge, 2011.

[13] Toby Cubitt, Laura Mancinska, David E. Roberson, Simone Severini, Dan Stahlke, Andreas Winter, Bounds on entanglement assisted source-channel coding via the Lovász theta number and its variants, IEEE Trans. Inform. Theory, 60 (2014), 73307344; arXiv: 1310.7120.

[14] Anton Dochtermann, Hom complexes and homotopy theory in the category of graphs, Europ. J. Combin., 30 (2009), 490-509; arXiv:math/0605275.

[15] Mohamed El-Zahar, Norbert Sauer, The chromatic number of the product of two 4-chromatic graphs is 4, Combinatorica, 5 (1985), 121-126.

[16] Paul Erdős, Chao Ko, Richard Rado, Intersection theorems for systems of finite sets, Quart. J. Math. Oxford Ser. (2), 12 (1961), 313-320.

[17] Paul Erdős, Robert J. McEliece, Herbert Taylor, Ramsey bounds for graph products, Pacific J. Math., 37 (1971), 45-46.

[18] Tobias Fritz, Resource convertibility and ordered commutative monoids, Math. Structures Comput. Sci., 27 (2017), 850-938.

[19] Chris Godsil, David E. Roberson, Robert Šamal, Simone Severini, Sabidussi versus Hedetniemi for three variations of the chromatic number, Combinatorica, 36 (2016), 395-415; arXiv: 1305.5545.

[20] Chris Godsil, David E. Roberson, Brendan Roomey, Robert Šamal, Antonios Varvitsiotis, Vector coloring the categorical product of graphs, Math. Program., 182 (2020), 275-314; arXiv:1801.08243.

[21] Robert E. Greenwood, Andrew M. Gleason, Combinatorial relations and chromatic graphs, Canad. J. Math., 7 (1955), 1-7.

[22] Willem Haemers, On some problems of Lovász concerning the Shannon capacity of a graph, IEEE Trans. Inform. Theory, 25 (1979), 231-232.

[23] Brandon Hanson, Giorgis Petridis, Refined estimates concerning sumsets contained in the roots of unity, Proc. London Math. Soc., (2020), https://doi.org/10.1112/plms.12322; arXiv:1905.09134.

[24] Xiaoyu He, Yuval Wigderson, Hedetniemi's conjecture is asympototically false, J. Combin.Theory, Ser. B, 146 (2021), 485-494; arXiv:1906.06783.

[25] Pavol Hell, An introduction to the category of graphs, Topics in graph theory (New York, 1977), Ann. New York Acad. Sci., 328, New York Acad. Sci., New York, 1979, 120-136,

[26] Pavol Hell, Jaroslav Nešetřil, Graphs and Homomorphisms, Oxford University Press, 2004. 
[27] Ron Holzman, private communication to Jeroen Zuiddam mentioned in [61] at $1: 28: 20$.

[28] Sihuang Hu, Itzhak Tamo, Ofer Shayevitz, A bound on the Shannon capacity via a linear programming variation, SIAM J. Discrete Math., 32 (2018), no. 3, 2229-2241.

[29] David Karger, Rajeev Motwani, Madhu Sudan, Approximate graph coloring by semidefinite programming, J. ACM, 45 (1998), 246-265.

[30] Ashik Mathew Kizhakkepallathu, Patric R. J. Östergård, Alexandru Popa, On the Shannon capacity of triangular graphs, Electron. J. Combin., 20(2) (2013), \#P27.

[31] Martin Kneser, Aufgabe 300, Jahresber. Deutsch., Math. Verein., 58 (1955) 27.

[32] László Lovász, Kneser's conjecture, chromatic number and homotopy, J. Combin. Theory, Ser. A, 25 (1978), no. 3, 319-324.

[33] László Lovász, On the Shannon capacity of a graph, IEEE Trans. Inform. Theory, 25 (1979), 1-7.

[34] László Lovász, Combinatorial Problems and Exercises, 2nd edition, Akadémiai Kiadó, Budapest and Elsevier Science Publishers, 1993.

[35] László Lovász, Graphs and Geometry, AMS, 2019.

[36] Robert J. McEliece, Edward C. Posner, Hide and seek, data storage and entropy, Ann. Math. Stat. (1971), 1706-1716.

[37] Jaroslav Nešetřil, Moshe Rosenfeld, I. Schur, C. E. Shannon and Ramsey numbers, a short story, Discrete Math., 229 (2001), 185-195.

[38] The Online Encyclopedia of Integer Sequences, https://oeis.org/A002496

[39] Wojciech Peisert, All self-complementary symmetric graphs, J. Algebra, 240 (2001), 209-229.

[40] Horst Sachs, Über selbstkomplementäre Graphen, Publ. Math. Debrecen, 9 (1962), 270-288.

[41] Norbert Sauer, Hedetniemi's conjecture-a survey, Discrete Math., 229 (2001), 261292.

[42] E. R. Scheinerman, D. H. Ullman, Fractional Graph Theory, Wiley-Interscience Series in Discrete Mathematics and Optimization, John Wiley and Sons, Chichester, 1997.

[43] Claude E. Shannon, The zero-capacity of a noisy channel, IRE Trans. Inform. Theory, 2 (1956), 8-19.

[44] Yaroslav Shitov, Counterexamples to Hedetniemi's conjecture, Ann. Math., 190 (2019), no. 2, 663-667; arXiv:1905.02167.

[45] Gábor Simonyi, Ambrus Zsbán, On topological relaxations of chromatic conjectures, Europ. J. Combin., 31 (2010), 2110-2119; arXiv:1002.4536 [math.CO].

[46] Daniel Stahlke, Python code to compute the Lovász, Schrijver, and Szegedy numbers for graphs, http://gist.github.com/dstahlke/6895643

[47] Volker Strassen. The asymptotic spectrum of tensors, J. Reine Angew. Math., 384 (1988), 102-152. 
[48] Claude Tardif, Multiplicative graphs and semi-lattice endomorphisms in the category of graphs, J. Combin. Theory, Ser. B, 95 (2005), 338-345

[49] Claude Tardif, Hedetniemi's conjecture, 40 years later, Graph Theory Notes N. Y., 54 (2008), 46-57.

[50] Claude Tardif, The chromatic number of the product of 14-chromatic graphs can be 13, manuscript (available on researchgate.net), 2020.

[51] Claude Tardif, Xuding Zhu, The level of nonmultiplicativity of graphs, Discrete Math., 244 (2002), 461-471.

[52] Claude Tardif, Xuding Zhu, A note on Hedetniemi's conjecture, Stahl's conjecture and the Poljak-Rödl function, Electron. J. Combin., 26(4) (2019), \#P4.32; arXiv: 1906.03748.

[53] Marcin Wrochna, Smaller counterexamples to Hedetniemi's conjecture; arXiv:2012.13558.

[54] Hong Zhang, Self-complementary symmetric graphs, J. Graph Theory, 16 (1992), $1-5$.

[55] Xuding Zhu, Star chromatic numbers and products of graphs, J. Graph Theory, 16 (1992), 557-569.

[56] Xuding Zhu, A survey on Hedetniemi's conjecture, Taiwanese J. Math., 2 (1998), $1-24$.

[57] Xuding Zhu, The fractional version of Hedetniemi's conjecture is true, European J. Combin., 32 (2011), 1168-1175.

[58] Xuding Zhu, A note on the Poljak-Rödl function, Electron. J. Combin., 27(3) (2020), \#P3.2; arXiv:1911.12015.

[59] Xuding Zhu, Relatively small counterexamples to Hedetniemi's conjecture, J. Combin. Theory, Ser. B, 146 (2021), 141-150; arXiv:2004.09028.

[60] Jeroen Zuiddam, The asymptotic spectrum of graphs and the Shannon capacity, Combinatorica, 39, 1173-1184; arXiv:1807.00169.

[61] Jeroen Zuiddam, Asymptotic spectra and their applications I, lecture at the Computer Science/Discrete Mathematics Seminar II, Institute for Advanced Study, Princeton, https://www . youtube. com/watch?v=1HwGSdikJxI 